\title{
EVOLUCIÓN DE LAS INVESTIGACIONES EN PALEONTOLOGÍA DE INVERTEBRADOS MARINOS EN LA REVISTA GEOLÓGICA DE AMÉRICA CENTRAL
}

\author{
EVOLUTION OF THE RESEARCH ON PALEONTOLOGY OF MARINE \\ INVERTEBRATES IN THE CENTRAL AMERICAN JOURNAL OF GEOLOGY
}

\author{
Teresita Aguilar
}

Centro de Investigaciones en Ciencias Geológicas (CICG), Ciudad Universitaria Rodrigo Facio, Universidad de Costa Rica. 125 m sur de los Condominios Cerros de Escazú, calle 28 Ave. 160 anatere.aguilar@gmail.com

(Recibido: 10/05/2014; aceptado: 12/08/2014)

\begin{abstract}
Fossil marine invertebrates are a very important group in Costa Rica and sourthen Central América, due to their abundance and high diversity and a strong relationship with geological processes, depositional environments and sometimes their restriction to short periods of time. Among the invertebrates Mollusks are dominant, followed by Arthropods, Echinodermata, Coelenterata, Porifera, Brachiopoda, Bryozoa and Annelida. These are showed in the scarcity of articles concerning to fossil invertebrates in the RGAC (13 in total), mainly about Mollusks (44 \%), primarily from Neogene (62\%) and mostly found in Costa Rica (85\%).

Keywords: Marine Invertebrate, Cretaceouss-Tertiary, Mollusks, Review

RESUMEN: Los invertebrados marinos fósiles son un grupo muy importante en Costa Rica y en el Sur de América Central, debido a su abundancia y gran diversidad, a su estrecha relación con los procesos geológicos, a los ambientes de depositación y eventualmente su restricción a ciertos períodos de tiempo. Entre los invertebrados los moluscos son los más abundantes, seguidos por los artropodos, equinodermos, Celenterados, Porifera, Brachiopoda, Bryozoa y Anelida. Esto se refleja en las escazas publicaciones sobre invertebrados fósiles en la RGAC (13 en total), las cuales tratan principalmente sobre Moluscos (44\%), fundamentalmente del Neógeno ( $62 \%$ ) y de Costa Rica ( $85 \%$ ).

Palabras clave: Invertebrados Marinos, Cretácico-Terciario, Moluscos, Revisiones
\end{abstract}




\section{INTRODUCCIÓN}

El conocimiento de los invertebrados marinos fósiles del Istmo Centroamericano y especialmente del área de Costa Rica y de Panamá, es de valor esencial para la Paleontología. La sola documentación del acervo de especies permite acercarnos más a una imágen real de la biodiversidad del pasado, que es la base para el entendimiento de la biodiversidad moderna de la región. Un segundo aspecto importante es la historia geológica del área, caracterizada por el desarrollo del Istmo Centroamericano al final del Cretácico y durante el Terciario, cerrándose completamente a finales del Neógeno (Fischer \& Aguilar, 1994). El cierre del Istmo provoca una evolución separada de las faunas marinas del Caribe y del Pacífico oriental después de un largo desarrollo en común con el área Paleocaribe. Finalmente, la formación y el crecimiento del Istmo originan cambios rápidos de los ambientes marinos asociados a sus costas, pasando de zonas batiales y abisales, a zonas neríticas y litorales y la consecuente colonización y desarrollo de faunas adaptadas a éstos ambientes. Los fósiles preservados representan la relación de las asociaciones marinas con los factores geológicos externos y reflejan la interacción entre cambios evolutivos, climáticos y ecológicos.

La influencia del Istmo como nuevo elemento paleogeográfico en la ecología, la distribución y la evolución de las faunas marinas empieza a notarse en el sur de América Central, en el transcurso del Mioceno. Es por eso que los invertebrados fósiles de ésa época son más abundantes, y su conocimiento es fundamental por ser la base del subsecuente desarrollo de las faunas de invertebrados del
Plioceno y Cuaternario del Neotrópico. Entre éstos fósiles destacan los moluscos bentónicos por el número de especies e indivíduos, por su preservación generalmente mejor que la de otros fósiles y por su registro ampliamente autóctono o/y parautóctono.

Los primeros y más completos estudios sobre invertebrados fósiles en la región, han sido realizados en Costa Rica y en la Zona del Canal de Panamá. Al inicio los investigadores fueron extranjeros que se interesaron en la región principalmente por la construcción del Canal de Panamá (Aguilar, 2009). Estos trabajos constituyeron monografías completas y publicaciones en revistas internacionales, en muchos casos de difícil acceso para los investigadores de los países de la región. Así, los trabajos de Gabb (1860, 1881 y 1889); Dall (1912); Hass (1942); Olsson (1922, 1942 ); Toula $(1909,1911)$ y Woodring $(1926$, 1957, 1959, 1964, 1970, 1973 у 1982), entre otros, constituyen descripciones detalladas de la fauna de invertebrados marinos, primordialmente moluscos, presentes en la región. Posteriormente, las investigaciones son realizadas por extranjeros que trabajaron especialmente en Costa Rica, en algunos casos relacionados con la Escuela Centroamericana de Geología y por extranjeros en colaboración con nacionales. Ejemplos de éstos trabajos son Carballo \& Fischer (1978); Fischer \& Franco (1979); Fischer (1985); Aguilar \& Fischer 1986); Woodring \& Malavassi (1961).

Con la creación de la Revista Geológica de América Central ( RGAC) en 1984, surgió un mecanismo accesible para que los nacionales y centroamericanos interesados pudieran realizar sus publicaciones y de esta forma dar a conocer 


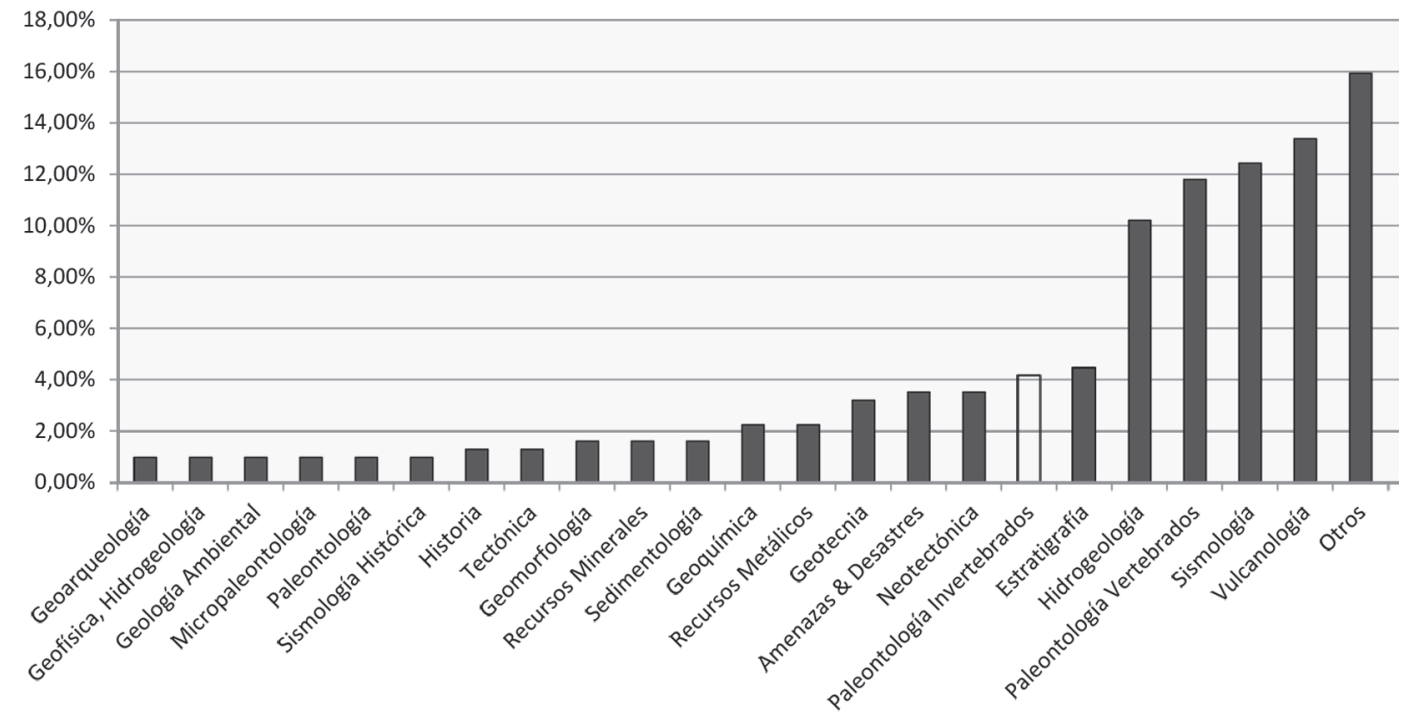

Fig. 1: Total de publicaciones por temas en la Revista Geológica de América Central.

los resultados de sus investigaciones. A pesar de eso, no abundan las publicaciones exclusivamente sobre invertebrados en la RGAC (13 en total) (Fig. 1), posiblemente porque no existió un desarrollo importante de la Paleontología en general, ni de los estudios sobre invertebrados en particular en la región, asociado a que no hubo un fuerte desarrollo en la exploración y explotación de recursos de origen sedimentario como el petróleo, así como a la existencia, hasta hace pocos años, de una carrera de Geología solamente en la Universidad de Costa Rica. Como consecuencia no se formaron especialistas en los diferentes grupos, lo cual queda demostrado por las pocas publicaciones.
Además, las condiciones geológicas y paleoecológicas de la región, principalmente a partir del Neógeno, favorecieron la preservación de faunas de moluscos, que son muy abundantes y diversos en esos ambientes, por lo que muchos de los paleontólogos interesados en la región se especializaron en éste grupo (Woodring W.P., Olsson A., Fischer R. y Aguilar T.).

Este hecho se refleja en que la mayoría de los trabajos publicados en la RGAC tratan sobre los moluscos (44\%), lo que está claramente relacionado a que los invertebrados marinos más abundantes en el registro estratigráfico de Costa Rica y de América Central pertenecen a los phylum Mollusca, seguidos por los Arthropoda (20\%) 


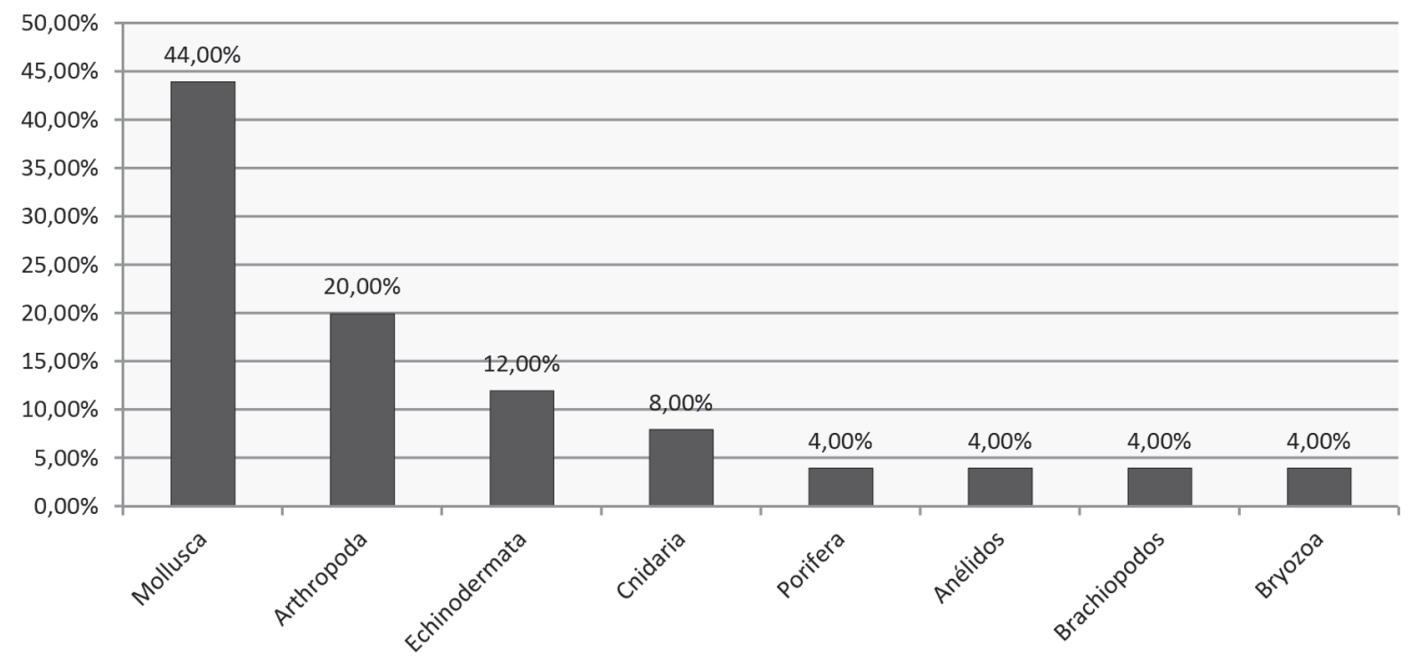

Fig. 2: Publicaciones de invertebrados por grupo taxonómico.

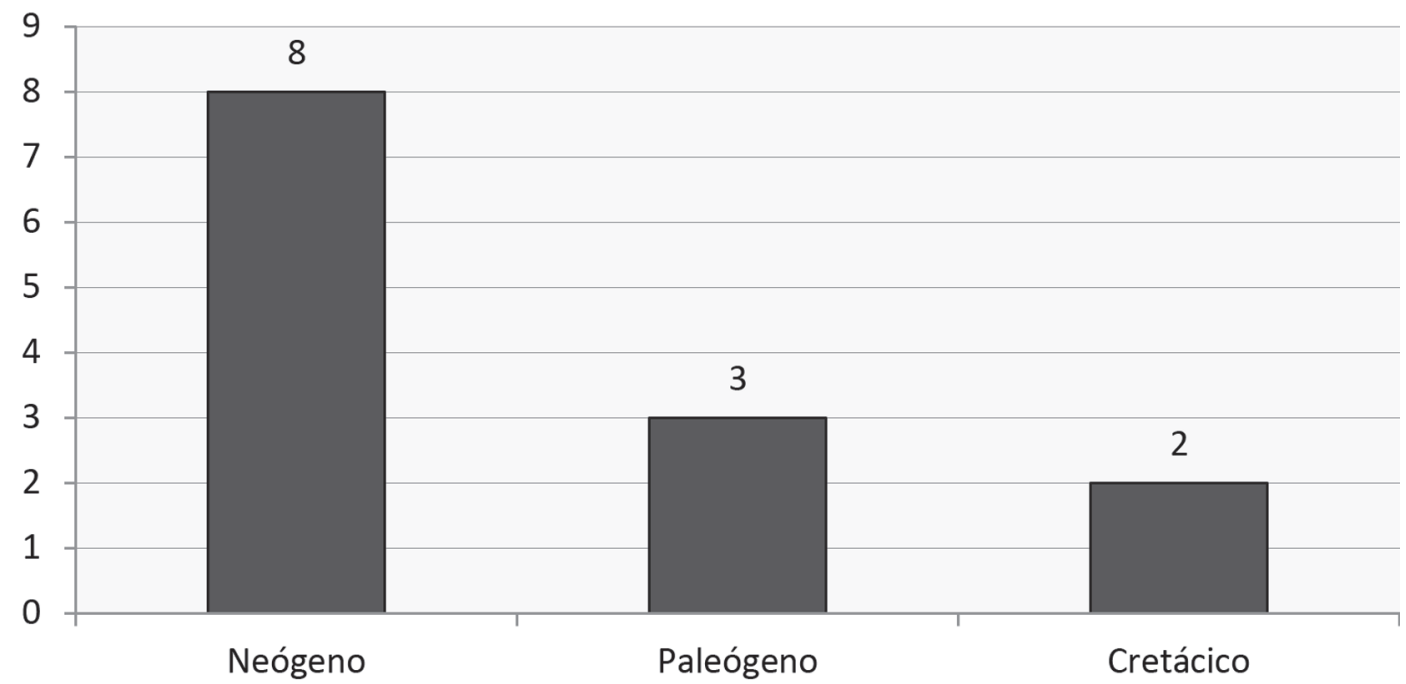

Fig. 3: Publicaciones de invertebrados por edad. 


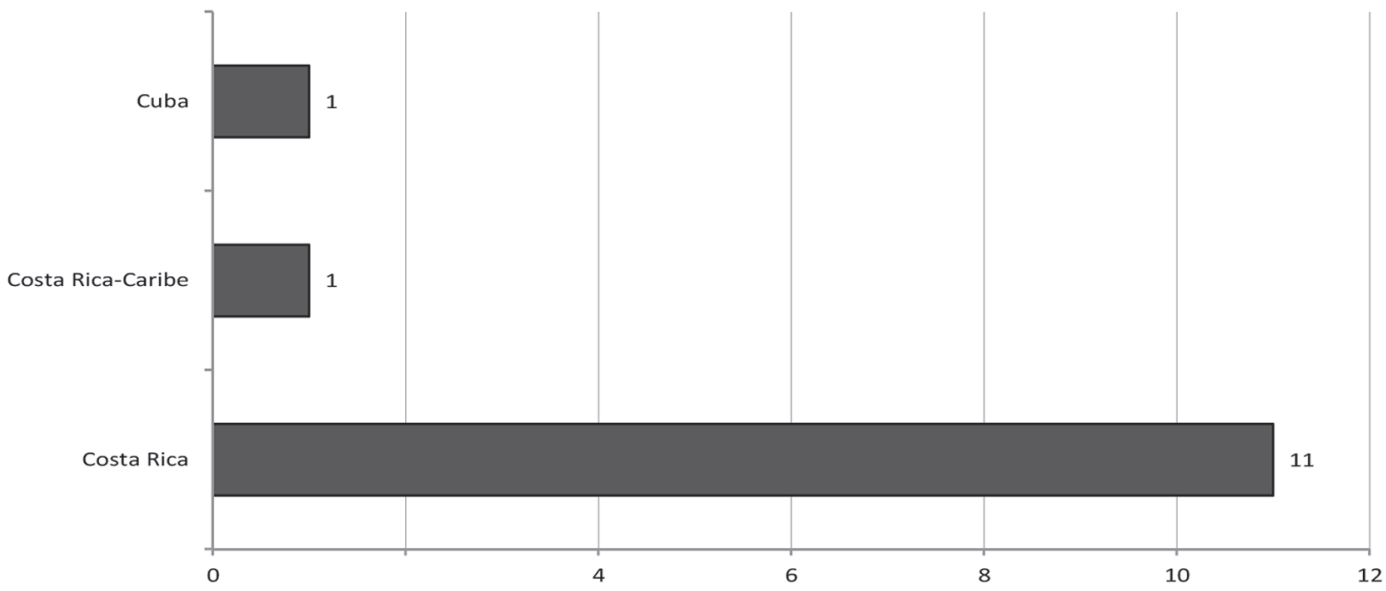

Fig. 4: Publicaciones de invertebrados por país de origen de la fauna.

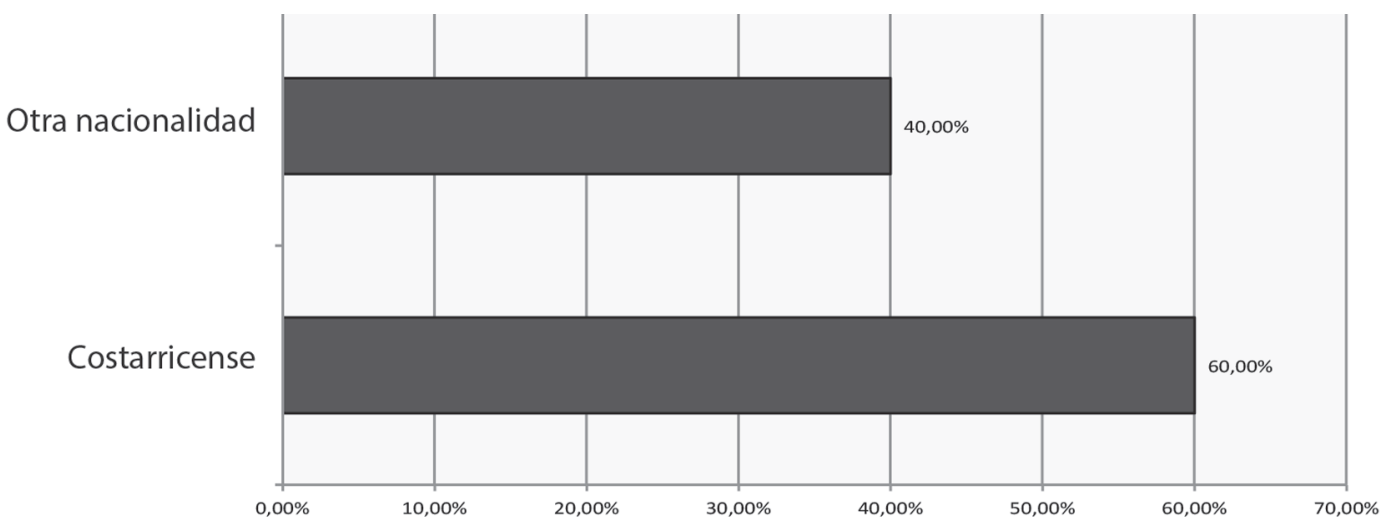

Fig. 5: Publicaciones de invertebrados por nacionalidad del o los autores.

y Echinodermata ( $8 \%$ ). Se encuentran también representantes de Coelenterata (Cnidaria $4 \%$ ), Porifera (4\%), Barchiopoda (4\%), Bryozoa (4 $\%$ ) y Annelida (4\%) pero en menor cantidad (Fig. 2).

Las asociaciones de invertebrados más abundantes y diversas se desarrollaron durante el Neógeno, por lo que las investigaciones que tratan sobre la descripción de éstas faunas, son más frecuentes para esta edad (Fig. 3).
La mayoría de trabajos tratan principalmente sobre faunas de Costa Rica (11), con algunas referencias a la región centroamericana, una se extiende al Caribe y una trata una fauna encontrada en Cuba (Fig. 4). Las publicaciones fueron hechas en su mayoría por costarricenses $(60 \%)$ o por costarricenses en colaboración con extranjeros (Fig. 5).

El aumento en las localidades estudiadas desde el punto de vista geológico, probablemente 
tendrá como resultado el hallazgo de más afloramientos con invertebrados fósiles, por lo que se hace necesario la preparación de especialistas en el reconocimeinto y clasificación de éstos fósiles y aprovechar su aplicabilidad en el establecimiento de los ambientes sedimentarios del pasado $\mathrm{y}$ cuando sea posible, de las edades de las rocas que los contienen con el fin de reflejar los resultados mediante publicaciones científicas.

\section{REFERENCIAS BIBLIOGRÁFICAS}

AGUILAR, T., 2009: Marine biodiversity of Costa Rica, Central America.- En: WEHRTMANN, I. S. \& CORTÉS, J. (eds.): Marine fossils.- Monographiae Biologicae, Springer Verlag, Germany: 81-94.

AGUILAR, T., \& FISCHER, R., 1986: Moluscos de la Formación Montezuma. PliocenoPleistoceno, Costa Rica.- Geologica et Palaeontologica, 20: 209-241.

CARBALLO, M.A. \& FISCHER, R., 1978: La Formación San Miguel (Mioceno, Costa Rica).- Inf. Semestral IGN (enero-junio): 45-144.

DALL, W. H., 1912: New species of fossil shells from Panamá and Costa Rica.- Smithsonian Misc. Coll. 59, 2(2977):1-10.

DALL, W. H., 1961: Miocene echinoids form the Valle Central, Costa Rica.- J. Paleont. 35(3):480-488.

HAAS, O., 1942: Miocene molluscs from Costa Rica.- J. Paleont. 16:307-316.

GABB, W. M., 1860: Description of some new species of Tertiary fossils from Chiriquí, Central America.- Proc. Acad. Nat. Sci. Philadelphia, 1860: 567-578.
GABB, W. M., 1881: Description of Caribbean Miocene fossils.- J. Acad. Natur. Sci. Philadelphia, 2(8): 337-348.

GABB, W. M., 1889: Descriptions of new species of fossils from the Pliocene claybeds between Limon and Moen, Costa Rica.- Acad. Natur. Sci. Philadelphia, (2), 8: 349-380.

FISCHER, R., 1985: La fauna de la Formación Turrúcares (Mioceno, Valle Central, Costa Rica).- Geologica et Palaeontologica, 19:191-225.

FISCHER, R., \& AGUILAR, T., 1994: Palaeontology of an evolving island arc.Profil, 7:391-400.

FISCHER, R., \& FRANCO, J. C., 1979: La Formación Coris (Mioceno; Valle Central, Costa Rica).- Inf. Semestral IGN (enerojunio): 15-71.

OLSSON, A. A., 1922: The Miocene of northern Costa Rica. With notes on its general stratigraphic relations.- Bull. Amer. Paleont. 9(39): 173-481.

OLSSON, A. A., 1942: Tertiary and Quarternary fossils from the Burica Peninsula of Panama and Costa Rica.- Bull. Amer. Paleont. 27(106): 1-106.

TOULA, F., 1909: Eine jungtertiaere Fauna von Gatun am Panama-Kanal.- Jahrbuch der Kaiserlich Königlichen Geologischen Reichsanstalt, 58: 673-760.

TOULA, F., 1911: Die jungtertiaere Fauna von Gatun am Panama-Kanal. II.Teil.- Jahrbuch der Kaiserlich Königlichen Geologischen Reichsanstalt, 61: 486-530. 
WOODRING, W. P., 1926: American Tertiary mollusks of the genus Clementia.- U.S. Geol. Surv.- Geol. Surv. Prof. Paper, 147C: $25-47$.

WOODRING, W. P., 1957: Geology and Paleontology of Canal Zone and adjoining parts of Panama. Geology and description of Tertiary molluscs (Gastropods: Trochidae to Turritellidae).- Geol. Surv. Prof. Paper, 306-A:1-145.

WOODRING, W. P., 1959: Geology and Paleontology of Canal Zone and adjoining parts of Panama. Description of Tertiary molluses (Gastropods: Vermetidae to Thaididae).- Geol. Surv. Prof. Paper, 306B:147-239.

WOODRING, W. P., 1964: Geology and Paleontology of Canal Zone and adjoining parts of Panama. Description of Tertiary molluscs (Gastropods: Columbellidae to Volutidae).- Geol. Surv. Prof. Paper, 306C: 241-297.

WOODRING, W. P., 1970: Geology and Paleontology of Canal Zone and adjoining parts of Panama. Description of
Tertiary molluscs (Gastropods: Eulimidae, Marginellidaeto Helminthoglyptidae).Geol. Surv. Prof. Paper, 306-D: 299-452.

WOODRING, W. P., 1973a: The Miocene Caribbean faunal province and its subprovinces.- Verh. Naturforsch. Ges. Basel, 84(1): 209-213.

WOODRING, W. P., 1973b: Geology and Paleontology of Canal Zone and adjoining parts of Panama. Description of Tertiary molluscs (Additions to gastropods, pelecypods: Nuculidae to Mallaeidae).- Geol. Surv. Prof. Paper, 306-E:453-539.

WOODRING, W. P., 1982: Geology and Paleontology of Canal Zone and adjoining parts of Panama. Description of Tertiary molluscs (Pelecypods: Propeamussidae to Cuspidariidae. Additions to families covered in P-306-E; Additions to Gastropods; Cephalopods).- Geol. Surv. Prof. Paper, 306-F: 541-759.

WOODRING, W. P., \& MALAVASSI , E., 1961: Miocene foraminifera, mollusks, and a barnacle from the Valle Central, Costa Rica.- J. Paleont. 35(3): 489-497. 
